\title{
Connections between relation algebras and cylindric algebras
}

\author{
Ian Hodkinson \\ Department of Computing, Imperial College London
}

\begin{abstract}
We give an informal description of a recursive representabilitypreserving reduction of relation algebras to cylindric algebras.
\end{abstract}

The final publication is available at

http://link. springer. com/chapter/10.1007\%2F978-3-319-24704-5_2

\section{Introduction}

Relation algebras form one of the principal algebraic approaches to binary relations. Introduced by Tarski in 1941 [9], their history actually goes back much further, to work of Peirce, Schröder, De Morgan, and even Boole. One of the key algebraic approaches to relations of higher arity is cylindric algebras, introduced by Tarski and his students Louise Chin and Frederick Thompson in the late 1940s.

Finding connections between relation algebras and cylindric algebras has been a prickly problem for a long time. There are a number of reasons why the problem might be of interest.

1. Algebraic logic generally seems to comprise a large number of formally different kinds of algebra - relation algebras, cylindric algebras, diagonal-free algebras, substitution algebras, polyadic (equality) algebras, and so on. Once one has proved a result for one kind of algebra, one is under some scientific obligation to try to prove it again for others.

Sometimes, doing this involves substantial technical innovation. But often, it can seem like merely copying out the old proof with minor modifications to take account of the different type of algebra. The core argument, often combinatorial in nature, remains the same. This leads in the direction of off-putting repetitive papers, allegations of 'salami slicing', and unpleasant subjective debates about how incremental a paper is.

In both cases, it would be valuable to have some reasonably general 'transfer theorems' allowing direct export of results from one kind of algebra to another. Indeed, such theorems might be more illuminating than just reformulating the same argument in slightly different terms.

2. A case in point is the 'negative' result that there is no algorithm to decide whether a finite relation algebra is representable $[1,2]$. The proof was complicated. Redoing it for cylindric algebras is even more complicated. A 'transfer 
result' would be very helpful here. It would snatch a 'positive' result from the jaws of negativity. (It is not my fault that problems are undecidable, but as one distinguished logician on the RAMiCS programme committee once told me, 'People get fed up with negative results.')

3. The question of connections between algebras of different arities (and perhaps varying in other features too) is of interest in its own right. It has a distinguished history, including work of Monk [8] and Maddux [5-7]. It raises intricate technical challenges.

4. Unlike first-order logic, algebraic logic is rather picky about arities. Usually there is a separate class of algebras for each arity (though some such as Craig have defined algebras comprising relations of multiple arities). So it would be nice to shed light on this separation and perhaps show it is less strict than appears.

5. Both relation algebras and cylindric algebras are listed on http://ramics2015. di.uminho.pt as in the scope of RAMiCS — so why not study their connections?

In this short note, we will attempt to give a gentle introduction to some work in this area. As a case study, we will focus on the problem mentioned in point 2 above: it is known to be undecidable whether a finite relation algebra is representable; can we use this result to show the same for finite $n$-dimensional cylindric algebras, for each finite $n \geq 3$ ? $^{1}$

The obvious approach is to find a recursive reduction of the first problem to the second. That is, we find a recursive function $f$ that, given a finite relation algebra $\mathcal{A}$, returns a finite $n$-dimensional cylindric algebra $f(\mathcal{A})$ that is representable when and only when $\mathcal{A}$ is representable. ${ }^{2}$ It would then of course follow that no algorithm could decide representability of $n$-dimensional cylindric algebras. For such an algorithm could be coupled with $f$ to provide an algorithm to decide representability of finite relation algebras, something that [1] assures us does not exist.

In section 3 below, we will recall briefly some earlier work on connections between relation algebras and cylindric algebras, and discuss the prospects for using it to construct such an $f$. Then, in section 4 , we explain informally a simplified form of the construction of $f$ from [3].

Since the full proofs are already in print, in this short note we will give only informal descriptions, not proofs. For clarity, we will make some simplifying assumptions. So: we will mostly restrict attention to the case of finite simple algebras. We will refrain from considering other algebras such as polyadic algebras and diagonal-free algebras. We will consider cylindric algebras only of finite

\footnotetext{
${ }^{1}$ We caution the reader that just because it appears 'harder' to represent cylindric algebras than relation algebras, it doesn't follow that the question of deciding whether a finite cylindric algebra is representable is harder than the corresponding question for relation algebras.

${ }^{2}$ In passing, we mention a converse problem: is there a recursive function $g$ that, given a finite $n$-dimensional cylindric algebra $\mathcal{C}$, returns a finite relation algebra $g(\mathcal{C})$ that is representable iff $\mathcal{C}$ is representable?
} 
dimensions $n \geq 3$. (The case $n<3$ is easy - representability is decidable in this case - and infinite-dimensional cylindric algebras take us in some sense outside the realm of finite algebras.)

\section{Definitions}

We recall the necessary basics. We adopt the standard convention that denotes the domain of an algebra $\mathcal{A}$ by $A$.

\section{$2.1 \quad$ Relation algebras}

A relation algebra is an algebra

$$
\mathcal{A}=(A,+,-, 0,1,1, \stackrel{\sim}{,}, ;),
$$

where $(A,+,-, 0,1)$ is a boolean algebra, called the boolean reduct of $\mathcal{A},\left(A, ;, 1^{\prime}\right)$ is a monoid, $`$ is a unary function on $A$, and $\mathcal{A}$ satisfies the Peircean law: $(a ; b)$. $c \neq 0 \Longleftrightarrow(\breve{a} ; c) \cdot b \neq 0 \Longleftrightarrow(c ; \breve{b}) \cdot a \neq 0$, for all $a, b, c \in A$, where $a \cdot b=-(-a+-b)$ (we will not use these properties in detail here).

We say that $\mathcal{A}$ is simple if $1 ; a ; 1=1$ for each non-zero $a \in A$, and finite if $A$ is finite. Mostly we will consider only finite simple relation algebras here. We warn the reader that for arbitrary relation algebras, some definitions and results below need to be modified, or may even fail.

Representations A (square) representation of $\mathcal{A}$ is a one-one map $h: A \rightarrow \wp\left(U^{2}\right)$, for some 'base' set $U$, such that for all $a, b \in A$,

1. $h(a+b)=h(a) \cup h(b)$

2. $h(-a)=U^{2} \backslash h(a)$

3. $h(0)=\emptyset$

4. $h(1)=U^{2}$

5. $h\left(1^{\prime}\right)=\{(x, x): x \in U\}$

6. $h(\breve{a})=\left\{(x, y) \in U^{2}:(y, x) \in h(a)\right\}$

7. $h(a ; b)=\left\{(x, y) \in U^{2}: \exists z((x, z) \in h(a) \wedge(z, y) \in h(b))\right\}$.

So $h$ represents each $a \in A$ as a binary relation on $U$, and the algebraic operations correspond via $h$ to 'concrete' operations on binary relations. Not every finite simple relation algebra is representable (i.e., has a representation). By $[2$, theorem 18.13], it is undecidable whether a finite simple relation algebra is representable.

Atoms, atomic relation algebras We can define a standard 'boolean' partial ordering $\leq$ on $A$ by $a \leq b$ iff $a+b=b$. An atom of $\mathcal{A}$ is a $\leq$-minimal non-zero element of $A$. We write $A t \mathcal{A}$ for the set of atoms of $\mathcal{A}$. We say that $\mathcal{A}$ is atomic if every non-zero element of $A$ lies $\leq$-above an atom. Every finite relation algebra is atomic. 
Atom structures and complex algebras By standard duality, an atomic relation algebra has an associated atom structure: a relational structure At $\mathcal{A}=$ $\left(A t \mathcal{A}, R_{1}, R_{\iota}, R_{;}\right)$, where $R_{1},=\left\{a \in A t \mathcal{A}: a \leq 1^{\prime}\right\}, R=\left\{(a, b) \in(A t \mathcal{A})^{2}:\right.$ $b \leq \breve{a}\}$, and $R_{;}=\left\{(a, b, c) \in(A t \mathcal{A})^{3}: c \leq a ; b\right\}$.

If $\mathcal{A}$ is finite, it is completely determined by At $\mathcal{A}$ up to isomorphism. Indeed, given any structure $\mathcal{S}=\left(S, R_{1}, R_{\triangleleft}, R_{;}\right)$of the signature of relation algebra atom structures, we can define its complex algebra $\mathfrak{C m} \mathcal{S}$ :

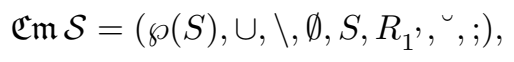

where, for $a, b \subseteq S, \breve{a}=\{s \in S: \exists t \in a(R \sim(t, s))\}$ and $a ; b=\{s \in S: \exists t \in$ $\left.a \exists u \in b\left(R_{;}(t, u, s)\right)\right\}$. Given suitable conditions on $\mathcal{S}$, this algebra $\mathfrak{C} \mathfrak{S} \mathcal{S}$ will be a relation algebra, and $\mathcal{S} \cong$ At $\mathfrak{C m} \mathcal{S}$. For finite relation algebras $\mathcal{A}$, we have $\mathcal{A} \cong \mathfrak{C m} \operatorname{At} \mathcal{A}$.

The atom structure of a finite relation algebra $\mathcal{A}$ is a good way to handle $\mathcal{A}$, since it is exponentially smaller. We can define particular finite relation algebras by specifying their atom structures.

Networks Another important concept to do with atoms and atom structures is that of network.

Given a finite simple relation algebra $\mathcal{A}$, an $\mathcal{A}$-network (over $N_{1}$ ) is a pair $N=\left(N_{1}, N_{2}\right)$, where $N_{1}$ is a set of 'nodes', and $N_{2}: N_{1} \times N_{1} \rightarrow A t \mathcal{A}$ is a 'labelling function' satisfying, for all $x, y, z \in N_{1}$,

1. $N_{2}(x, x) \leq 1$,

2. $N_{2}(x, y)=N_{2}(y, x)^{\smile}$ (we note that in relation algebras, ${ }^{\triangleleft}$ takes atoms to atoms),

3. $N_{2}(x, y) \leq N_{2}(x, z) ; N_{2}(z, y)$.

Frequently we drop the indices 1,2 , deducing them by context.

Networks arise from parts of representations. The key observation here is that if $h: A \rightarrow \wp\left(U^{2}\right)$ is a representation of the (finite simple) relation algebra $\mathcal{A}$, then for each $u, v \in U$ there is a unique atom $a \in A$ such that $(u, v) \in h(a)$ (it is an exercise to show that this atom exists and is unique). Writing this atom as $\lambda(u, v)$, so that $\lambda: U^{2} \rightarrow A t \mathcal{A}$ is a function, it can be checked that for each $X \subseteq U$, the pair

$$
\left(X, \lambda \uparrow X^{2}\right)
$$

is an $\mathcal{A}$-network. The 'whole' network $N=(U, \lambda)$ satisfies an additional 'saturation' condition

4. for each $x, y \in N$ and atoms $a, b \in A$, if $N(x, y) \leq a ; b$, then there exists $z \in N$ with $N(x, z)=a$ and $N(z, y)=b$.

Conversely, any 'saturated' $\mathcal{A}$-network $N$ satisfying condition 4 can be viewed as a representation $h$ of $\mathcal{A}$ via $h(a)=\{(x, y) \in N: N(x, y) \leq a\}$, for each $a \in A$. Or almost. This $h$ need not respect 1', since $N^{\prime}(x, y) \leq 1$ ' does not imply $x=y$. We say that $h$ is a loose representation of $\mathcal{A}$. To get a pukka representation, we need to factor out by the equivalence relation $h\left(1^{\prime}\right)$ on $N$. 
We end with some notation that will be useful. For $\mathcal{A}$-networks $N=\left(N_{1}, N_{2}\right)$ and $M=\left(M_{1}, M_{2}\right)$, and any objects $i_{1}, \ldots, i_{k}$, we write

$$
N={ }_{i_{1}, \ldots, i_{k}} M
$$

if $N_{1} \backslash\left\{i_{1}, \ldots, i_{k}\right\}=M_{1} \backslash\left\{i_{1}, \ldots, i_{k}\right\}=I$, say, and $N(i, j)=M(i, j)$ for all $i, j \in I$. That is, $M$ and $N$ agree off of $\left\{i_{1}, \ldots, i_{k}\right\}$.

\subsection{Cylindric algebras}

Just as relation algebras 'algebraise' binary relations, so cylindric algebras algebraise relations of higher arities. From now on, fix some finite dimension (or arity) $n \geq 3$. An $n$-dimensional cylindric algebra is an algebra

$$
\mathcal{C}=\left(C,+,-, 0,1, \mathrm{~d}_{i j}, \mathrm{c}_{i}: i, j<n\right),
$$

where $(C,+,-, 0,1)$ is a boolean algebra as before, the $\mathrm{d}_{i j}$ are constants, and the $c_{i}$ are unary functions on $C$, satisfying certain equations not needed here. The algebra $\mathcal{C}$ is said to be finite if $C$ is finite, and simple if $\mathrm{c}_{0} \mathrm{c}_{1} \cdots \mathrm{c}_{n-1} a=1$ for each non-zero $a \in C$.

A (square) representation of $\mathcal{C}$ is a map $h: C \rightarrow \wp\left(U^{n}\right)$, for some base set $U$, respecting the boolean operations as before, and with

1. $h\left(\mathrm{~d}_{i j}\right)=\left\{\left(x_{0}, \ldots, x_{n-1}\right) \in U^{n}: x_{i}=x_{j}\right\}$

2. $h\left(c_{i} a\right)=\left\{\left(x_{0}, \ldots, x_{n-1}\right) \in U^{n}: \exists\left(y_{0}, \ldots, y_{n-1}\right) \in h(a)\left(x_{j}=y_{j}\right.\right.$ for each $j \in n \backslash\{i\})\}$

for each $i, j<n$ (we identify $n$ with $\{0,1, \ldots, n-1\}$ ) and each $a \in C$. So this time, each element of the algebra is 'represented' as an $n$-ary relation on $U$. The elements of $C$ are like first-order formulas written with variables $x_{0}, \ldots, x_{n-1}$; $\mathrm{d}_{i j}$ is like $x_{i}=x_{j}$, and $c_{i} a$ is like $\exists x_{i} a$. Again, not every $n$-dimensional finite simple cylindric algebra $(n \geq 3)$ is representable.

Atoms and atomic cylindric algebras are defined as for relation algebras. The atom structure of an atomic $n$-dimensional cylindric algebra $\mathcal{C}$ as above is the structure

$$
\operatorname{AtC}=\left(A t \mathcal{C}, R_{\mathrm{d}_{i j}}, R_{\mathrm{c}_{i}}: i, j<n\right),
$$

where $R_{\mathrm{d}_{i j}}=\left\{a \in A t \mathcal{C}: a \leq \mathrm{d}_{i j}\right\}$ and $R_{\mathrm{c}_{i}}=\left\{(a, b) \in(A t \mathcal{C})^{2}: b \leq \mathrm{c}_{i} a\right\}$. A structure $\mathcal{S}=\left(S, R_{\mathrm{d}_{i j}}, R_{\mathrm{c}_{i}}: i, j<n\right)$ in this signature is called a $n$-dimensional cylindric-type atom structure. Again, we can form its complex algebra:

$$
\mathfrak{C m} \mathcal{S}=\left(\wp(S), \cup, \backslash, \emptyset, S, R_{\mathrm{d}_{i j}}, c_{i}: i, j<n\right),
$$

where $\mathrm{c}_{i} X=\left\{y \in S: R_{\mathrm{c}_{i}}(x, y)\right.$ for some $\left.x \in X\right\}$, for each $i<n$ and $X \subseteq S$. Under favourable conditions, $\mathfrak{C m} \mathcal{S}$ will be an $n$-dimensional cylindric algebra, and again we have $\mathcal{S} \cong \operatorname{At} \mathfrak{C m} \mathcal{S}$, and $\mathcal{C} \cong \mathfrak{C m}$ At $\mathcal{C}$ for each finite cylindric algebra $\mathcal{C}$.

One can also define networks for cylindric algebras, analogously to the relation algebra case. 


\section{Earlier work}

Now let us review some earlier work connecting relation algebras and cylindric algebras. We confine ourselves to the most relevant topics. For a far more thorough survey, see [7].

\subsection{Monk}

In [8], Monk gave a method of turning an arbitrary relation algebra $\mathcal{A}$ into a 3 -dimensional cylindric algebra $\mathcal{C}$, preserving representability both ways - that is, $\mathcal{A}$ is representable iff $\mathcal{C}$ is representable.

[8, p.63] states that the idea is due to Lyndon. [8, p.81] adds that 'This description occurs in a letter from Lyndon to Thompson dated May, 1949... in this letter he restricts himself to the case of proper relation algebras.' For our purposes, we can take a proper relation algebra to be a representable one; so by extending the construction to arbitrary relation algebras, Monk made a considerable advance. [8, p.81] also states that reference to the embedding has occurred in several places. The earliest of them is [4].

The construction is important, but rather complicated, and I'm reluctant to summarise it for fear of misrepresentation. However, a related idea is to regard the elements of a relation algebra $\mathcal{A}$ as binary relation symbols, consider the set of all first-order formulas using these symbols and written with only the variables $x_{0}, x_{1}, x_{2}$, and quotient it out by a certain equivalence relation (actually a congruence) suggested by the relation algebra operations. For example, $\exists x_{2}\left(a\left(x_{0}, x_{2}\right) \wedge b\left(x_{2}, x_{1}\right)\right)$ (where $a, b \in A$ ) would be equivalent to $(a ; b)\left(x_{0}, x_{1}\right)$. If done properly, the congruence classes form a 3 -dimensional cylindric algebra that is representable just when $\mathcal{A}$ is representable. For more on this, and much else, see [10].

For finite relation algebras, Monk's construction is recursive, and it follows by Turing reduction that representability of finite 3-dimensional cylindric algebras is undecidable.

Monk does not give any construction in dimensions higher than 3. For this, we need to pass to work of Maddux.

\subsection{Maddux: cylindric bases}

In a number of publications, including notably [5,6] and the survey [7], Maddux gave a new way of constructing cylindric algebras of any dimension from atomic relation algebras, using sets of networks called cylindric bases. We will continue to simplify things by restricting consideration to finite simple relation algebras. For these, in dimension 3, Maddux's construction reproduces Monk's construction up to isomorphism. We will go into some detail about it, since we need it later. 
Idea Let us try to motivate the idea of cylindric basis. Suppose we are given a representation $h: A \rightarrow \wp\left(U^{2}\right)$ of a (finite simple) relation algebra $\mathcal{A}$. Recall from formula (1) in section 2.1 that a subset of the base set $U$ can be viewed as an $\mathcal{A}$-network. We can make this a little tighter by considering maps instead of subsets.

Definition 1. Let $N=\left(N_{1}, N_{2}\right)$ be an $\mathcal{A}$-network, and $h: A \rightarrow \wp\left(U^{2}\right)$ a representation of $\mathcal{A}$ with base $U$.

1. A partial map $f: N_{1} \rightarrow U$ is said to be a partial embedding of $N$ into $h$ if $(f(x), f(y)) \in h\left(N_{2}(x, y)\right)$ for all $x, y \in \operatorname{dom}(f)$.

2. We say that $f$ is a total embedding, or just an embedding, if $\operatorname{dom}(f)=N_{1}$.

3. We also say that $N$ embeds homogeneously into $h$ if every partial embedding of $N$ into $h$ extends to a total one.

Recall that $n \geq 3$ is our fixed finite dimension. For any $n$-tuple $\left(u_{0}, \ldots\right.$, $\left.u_{n-1}\right) \in U^{n}$, we can form an $\mathcal{A}$-network

$$
N_{\left(u_{0}, \ldots, u_{n-1}\right)}=(n, \nu)
$$

whose set of nodes is $n=\{0,1, \ldots, n-1\}$, where for each $i, j<n$, the label $\nu(i, j)$ is the unique atom $a$ of $\mathcal{A}$ with $\left(u_{i}, u_{j}\right) \in h(a)$ — that is, $\nu(i, j)=\lambda\left(u_{i}, u_{j}\right)$ in our earlier notation. Manifestly, the map $\left(i \mapsto u_{i}\right)_{i<n}$ is an embedding of $N_{\left(u_{0}, \ldots, u_{n-1}\right)}$ into $h$. In model-theoretic terms, $N_{\left(u_{0}, \ldots, u_{n-1}\right)}$ describes the atomic type of the tuple $\left(u_{0}, \ldots, u_{n-1}\right)$ in the representation.

Let $\mathcal{N}_{n}(\mathcal{A})$ denote the set of all $\mathcal{A}$-networks whose set of nodes is $n$. Each network $N \in \mathcal{N}_{n}(\mathcal{A})$ defines a (possibly empty) n-ary relation on $U$, namely

$$
\left\{\left(u_{0}, \ldots, u_{n-1}\right) \in U^{n}: N_{\left(u_{0}, \ldots, u_{n-1}\right)}=N\right\}
$$

This is the set of $n$-tuples onto which we can embed $N$. See figure 1 in the case $n=3$.

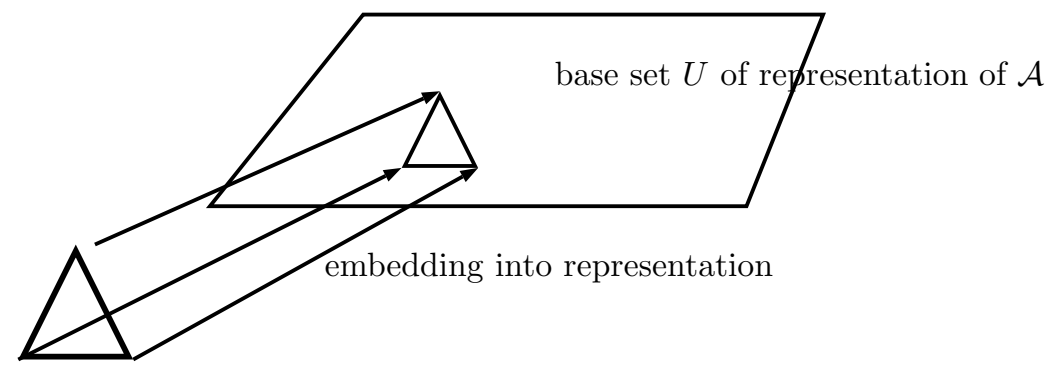

3-dimensional network

Fig. 1. Network embedding into representation 
In view of this, can we make an atomic $n$-dimensional cylindric algebra whose atoms are networks in $\mathcal{N}_{n}(\mathcal{A})$, whose arbitrary elements are subsets of $\mathcal{N}_{n}(\mathcal{A})$, and which in the above case is representable over the base set $U$ ?

Well, we would need to know exactly which networks embed into $h$, so that they arise as some $N_{\left(u_{0}, \ldots, u_{n-1}\right)}$. This might depend on the choice of the representation $h$ - and when $\mathcal{A}$ is not representable, there is no such $h$ ! Remember that our reduction map $f$ should deliver a cylindric algebra $f(\mathcal{A})$ given any finite simple relation algebra $\mathcal{A}$, representable or not. So we will need to 'guess' a suitable set - $B$, say — of networks to use.

But this is only the start of it. We also have to take account of the cylindric algebra operations.

The constants $\mathrm{d}_{i j}$ are easy to handle. In a representation of the cylindric algebra, we must interpret $\mathrm{d}_{i j}$ as $\left\{\left(u_{0}, \ldots, u_{n-1}\right) \in U^{n}: u_{i}=u_{j}\right\}$. But this is just $\left\{\left(u_{0}, \ldots, u_{n-1}\right) \in U^{n}: N_{\left(u_{0}, \ldots, u_{n-1}\right)}(i, j) \leq 1^{\prime}\right\}$. So we could let

$$
\mathrm{d}_{i j}=\left\{N \in B: N(i, j) \leq 1^{\prime}\right\}
$$

for each $i, j<n$.

The 'cylindrifiers' $c_{i}$ are a little harder. Plainly, if $\left(u_{0}, \ldots, u_{n-1}\right),\left(v_{0}, \ldots, v_{n}\right) \in$ $U^{n}, i<n$, and $u_{j}=v_{j}$ for each $j \in n \backslash\{i\}$, then $N_{\left(u_{0}, \ldots, u_{n-1}\right)}={ }_{i} N_{\left(v_{0}, \ldots, v_{n-1}\right)}$. For any relation $r$ in our putative cylindric algebra that holds on $\left(u_{0}, \ldots, u_{n-1}\right)$, the relation $\mathrm{c}_{i} r$ must hold on $\left(v_{0}, \ldots, v_{n-1}\right)$. So we could let

$$
\mathrm{c}_{i} N=\left\{M \in B: M={ }_{i} N\right\}
$$

for each $N \in B$. The generalisation to sets of networks is easy.

Notice that these definitions are dependent on $B$ but independent of any representation of $\mathcal{A}$ - they make sense even if $\mathcal{A}$ is not representable.

But for a correct representation of our hoped-for cylindric algebra, for every sequence $\left(u_{0}, \ldots, u_{n-1}\right) \in U^{n}$ with $\left.N_{\left(u_{0}, \ldots, u_{n-1}\right.}\right)=N$, and every $M \in B$ with $M={ }_{i} N$, there must be a sequence $\left(v_{0}, \ldots, v_{n}\right) \in U^{n}$ with $u_{j}=v_{j}$ for each $j \in n \backslash\{i\}$, and $N_{\left(v_{0}, \ldots, v_{n-1}\right)}=M$. This boils down to saying that each $N \in B$ embeds homogeneously into $h$.

This does turn out to be the case when $n=3$ and $B=N_{3}(\mathcal{A})$. But for higher dimensions, it is problematic, as we will now see.

Cylindric bases Let us be a little more formal. An $n$-dimensional cylindrical basis of a finite simple relation algebra $\mathcal{A}$ is a non-empty subset $B \subseteq \mathcal{N}_{n}(\mathcal{A})$ satisfying $^{3}$

1. For each $N \in B, i, j<n, k \in n \backslash\{i, j\}$, and atoms $a, b \in A t \mathcal{A}$, if $N(i, j) \leq$ $a$; $b$, then there exists $N^{\prime} \in B$ with $N^{\prime}={ }_{k} N, N^{\prime}(i, k)=a$, and $N^{\prime}(k, j)=b$.

2. If $N, M \in B, i, j<n$, and $N={ }_{i j} M$, then there is $P \in B$ with $N={ }_{i} P={ }_{j}$ $M$.

3 The definition we give here is not the same as Maddux's definition in (e.g.,) [6, definition 4], but it is equivalent to it for finite simple relation algebras. See, e.g., [2, lemma 12.36]. 
We will not use the details of this definition, but we do point the reader's attention to the similarity of clause 1 to our earlier saturation condition for $\mathcal{A}$ networks. The salient facts about cylindric bases are as follows.

1. We can view an $n$-dimensional cylindric basis $B$ as an $n$-dimensional cylindrictype atom structure

$$
\mathcal{B}=\left(B, R_{\mathrm{d}_{i j}}, R_{\mathrm{c}_{i}}: i, j<n\right),
$$

where $R_{\mathrm{d}_{i j}}=\left\{N \in B: N(i, j) \leq 1^{\prime}\right\}$ and $R_{\mathrm{c}_{i}}=\left\{(N, M) \in B^{2}: N={ }_{i} M\right\}$ — that is, $R_{\mathrm{c}_{i}}$ is just $={ }_{i}$. This is as suggested above. We write $B$ for the basis and $\mathcal{B}$ for the corresponding atom structure. The definition of cylindric basis ensures that $\mathfrak{C m} \mathcal{B}$ is always an $n$-dimensional cylindric algebra, with atom structure isomorphic to $\mathcal{B}$. The map $B \mapsto \mathfrak{C m} \mathcal{B}$ is recursive.

2. In dimension 3:

- Every finite simple relation algebra $\mathcal{A}$ has a 3 -dimensional cylindric basis. The set $\mathcal{N}_{3}(\mathcal{A})$ is one such, and it's the only one, actually.

- For $B=\mathcal{N}_{3}(\mathcal{A})$, the complex algebra $\mathfrak{C m} \mathcal{B}$ is a 3-dimensional cylindric algebra isomorphic to what Monk's construction gives.

- $\mathfrak{C} \mathfrak{m} \mathcal{B}$ is representable iff $\mathcal{A}$ is representable as a relation algebra. Here is the gist of the proof. For $\Leftarrow$, we can read off a representation of $\mathfrak{C m} \mathcal{B}$ from any representation of $\mathcal{A}$, because the relation algebra operations are strong enough to ensure that all networks in $\mathcal{N}_{3}(\mathcal{A})$ embed homogeneously into any representation of $\mathcal{A}$.

More formally, if $h: A \rightarrow \wp\left(U^{2}\right)$ is a representation of $\mathcal{A}$, then define a representation $h^{*}: \wp(B) \rightarrow \wp\left(U^{3}\right)$ of $\mathfrak{C} \mathfrak{m} \mathcal{B}$ by

$$
\left.h^{*}(X)=\left\{\left(u_{0}, u_{1}, u_{2}\right) \in U^{3}: N_{\left(u_{0}, u_{1}, u_{2}\right)} \in X\right)\right\}
$$

for each $X \subseteq B$, using the notation $N_{\left(u_{0}, \ldots, u_{n-1}\right)}$ introduced above. For $\Rightarrow, \mathcal{A}$ is a subalgebra of the (neat) relation algebra reduct of $\mathfrak{C m} \mathcal{B}$ obtained by restricting to its '2-dimensional elements'. Taking relation algebra reducts preserves representability. For more details, see [7, §4].

3. However, for dimensions $n>3$ :

- $\mathcal{N}_{n}(\mathcal{A})$ may not be an $n$-dimensional cylindric basis.

- For $n \geq 5$, not every atomic relation algebra $\mathcal{A}$ has any $n$-dimensional cylindric basis at all.

- Even when $\mathcal{A}$ does have an $n$-dimensional cylindric basis, say $B$, it may be that $\mathcal{A}$ is representable but $\mathfrak{C} \mathfrak{m} \mathcal{B}$ is not (though it will be an $n$ dimensional cylindric algebra). The 'reason' is that not every network in $B$ need embed (at all, or homogeneously) into a representation of $\mathcal{A}$. Examples can be found in [6, pp. 960-961] and [7, p. 389].

- It is true that if $\mathcal{A}$ has a cylindric basis $B$ and $\mathfrak{C m} \mathcal{B}$ is representable, then $\mathcal{A}$ is representable, as again it is a subalgebra of the relation algebra reduct of $\mathfrak{C m} \mathcal{B}$. But for a reduction, this is not enough.

So, while excellent in dimension 3 and very important in general, cylindric bases do not suit our purposes in higher dimensions than 3. 


\section{Reduction in arbitrary dimensions}

Recall that we wish to find a recursive construction of an $n$-dimensional cylindric algebra from an arbitrary finite simple relation algebra, and the construction should preserve representability both ways.

The constructions of Monk and Maddux do not achieve this aim in higher dimensions, but they do in dimension 3. We can learn from this.

An $n$-dimensional cylindric basis uses ' $n$-dimensional' networks with base set $n$. All pairs $(i, j)$ of nodes $i, j<n$ are 'labelled' with atoms. In dimensions higher than 3 , this can cause a mismatch between the kind of networks that exist in the basis and those that embed (homogeneously) into a representation of $\mathcal{A}$. But in dimension 3, there is no mismatch at all, because the relation algebra operations 'control' exactly which 3-dimensional networks embed in a representation (namely, all of them do), and moreover they ensure that every network embeds homogeneously as well.

So, let us try to devise a new kind of ' $n$-dimensional network' — one based on the 3-dimensional networks that work so well, without adding any extra higherdimensional structure from the relation algebra point of view. One can have all sorts of ideas about how to do this (believe me), but they often fail, because potentially fatal higher-dimensional information is smuggled in.

\subsection{Motivation from representations}

It can help to think in terms of representations. Given a representation $h: A \rightarrow$ $\wp\left(U^{2}\right)$ of $\mathcal{A}$, an $n$-tuple $\left(u_{0}, \ldots, u_{n-1}\right)$ of elements of $U$ 'sees' what the representation says about its points - that is, the collection of atoms $\lambda\left(u_{i}, u_{j}\right)$ associated with pairs of points from the tuple. This gives it information on the mutual relationships of up to $n$ points of the representation. This is dangerous, for the above-mentioned reasons. The challenge we face is to limit this information to groups of at most three points, while still having all of $u_{0}, \ldots, u_{n-1}$ around.

So we consider a new kind of structure. Let $V$ be a set. Suppose that for each subset $S \subseteq V$ of cardinality exactly $n-3$, we have a representation $h_{S}: A \rightarrow$ $\wp\left((V \backslash S)^{2}\right)$ of $\mathcal{A}$ on the base $V \backslash S$. Here, $S$ is a sort of 'black hole', carrying no information inside it. There need be no correlation whatever between the $h_{S}$, as $S$ varies.

Extending our earlier notation, for $u, v \in V \backslash S$ we write $\lambda_{S}(u, v)$ for the unique atom $a \in A t \mathcal{A}$ such that $(u, v) \in h_{S}(a)$.

Now, given an $n$-tuple $\left(u_{0}, \ldots, u_{n-1}\right)$ of elements of $V$, the only information from $\mathcal{A}$ that $\left(u_{0}, \ldots, u_{n-1}\right)$ can 'see' is the collection of atoms

$$
\left\langle\lambda_{S}\left(u_{i}, u_{j}\right): S \subseteq\left\{u_{0}, \ldots, u_{n-1}\right\},|S|=n-3, i, j<n, u_{i}, u_{j} \notin S\right\rangle .
$$

Crucially, only 3-dimensional information (from at most three points) about any one representation $h_{S}$ is now visible to $\left(u_{0}, \ldots, u_{n-1}\right)$. This is because at most three points of $u_{0}, \ldots, u_{n-1}$ can lie outside each $S$ in (2). Moreover, the cylindric algebra operations cannot be used to garner higher-dimensional information 
about $h_{S}$. For that would involve 'moving' a point $u_{i} \in S$ to a point outside $S$, using a c $c_{i}$. But then, $S$ is no longer a subset of the points in the resulting $n$-tuple, so (see (2)) no information about $h_{S}$ is available to it at all.

\subsection{Holograms}

So we wish to devise a new kind of $n$-dimensional network embodying the information in (2) above. The network will become an atom of our final cylindric algebra that will 'hold' on $\left(u_{0}, \ldots, u_{n-1}\right)$.

But the definition of the new network cannot use any representation, since $\mathcal{A}$ may not have a representation!

So we simply throw in any 3-dimensional networks, subject only to identity constraints. We call our new-style network a hologram, since it incorporates many different 3-dimensional 'views'.

Definition 2. Let $\sim$ be an equivalence relation on $n$. Write $H(\sim)$ for the set of all subsets $X \subseteq n$ such that $n \backslash X$ is the union of exactly $(n-3) \sim$-classes. Quite possibly, $H(\sim)=\emptyset$.

For a finite simple relation algebra $\mathcal{A}$, an (n-dimensional) hologram (over $\mathcal{A}$ ) is a family

$$
\eta=\left(\sim, N_{X}: X \in H(\sim)\right),
$$

where $\sim$ is an equivalence relation on $n$, each $N_{X}$ is an $\mathcal{A}$-network whose set of nodes is $X$, and for each $X \in H(\sim)$ and $i, j \in X$, if $i \sim j$ then $N_{X}(i, j) \leq 1$ '.

Example 1. In terms of the sketch in section 4.1 with $V$ and the $h_{S}$ and $\lambda_{S}$, a hologram $\eta=\left(\sim, N_{X}: X \in H(\sim)\right)$ will 'hold' on $\left(u_{0}, \ldots, u_{n-1}\right) \in V^{n}$ as per (2) iff:

H1 For each $i, j<n$ we have $u_{i}=u_{j}$ iff $i \sim j$.

H2 For each $X \in H(\sim)$, if $S=\left\{u_{k}: k \in n \backslash X\right\}$, then $N_{X}(i, j)=\lambda_{S}\left(u_{i}, u_{j}\right)$ for each $i, j \in X$. That is, the map $\left(i \mapsto u_{i}: i \in X\right)$ is an embedding of $N_{X}$ into $h_{S}$.

Note that if $X \in H(\sim)$, the set $n \backslash X$ is the union of exactly $n-3 \sim$-classes, and so no element of $X$ is $\sim$-equivalent to any element of $n \backslash X$. So by H1, the set $S$ in H2 has size $n-3$ and $u_{i} \notin S$ for each $i \in X$. Hence, H2 makes sense. Also note that for $i, j \in X$ we have $i \sim j \Rightarrow u_{i}=u_{j} \Rightarrow N_{X}(i, j)=\lambda_{S}\left(u_{i}, u_{j}\right) \leq 1$, which is consistent with definition 2 .

\subsection{Atom structure from holograms}

Let $M$ be the set of all ( $n$-dimensional) holograms. We wish to form an $n$ dimensional cylindric-type atom structure $\mathcal{M}=\left(M, R_{\mathrm{d}_{i j}}, R_{\mathrm{c}_{i}}: i, j<n\right)$. From $\mathrm{H} 1$ above, it is clear that we should define

$$
R_{\mathrm{d}_{i j}}=\left\{\left(\sim, N_{X}: X \in H(\sim)\right) \in M: i \sim j\right\} .
$$



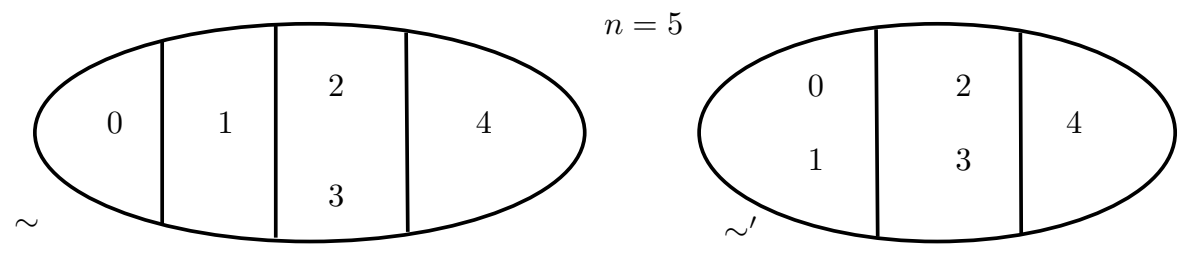

Fig. 2. Equivalence relations $\sim, \sim^{\prime}$ on 5

But what about $R_{\mathrm{c}_{i}}$ ? For inspiration, we consider again the picture in section 4.1. Suppose we have two tuples $\left(u_{0}, \ldots, u_{n-1}\right)$ and $\left(v_{0}, \ldots, v_{n-1}\right)$ in $V^{n}$, with $u_{j}=v_{j}$ for all $j \in n \backslash\{i\}$. What is the connection between the holograms $\left(\sim, N_{X}: X \in H(\sim)\right)$ and $\left(\sim^{\prime}, N_{X}^{\prime}: X \in H\left(\sim^{\prime}\right)\right)$ that 'hold' in the sense of example 1 on $\left(u_{0}, \ldots, u_{n-1}\right)$ and $\left(v_{0}, \ldots, v_{n-1}\right)$, respectively?

Well, we certainly have $j \sim k$ iff $j \sim^{\prime} k$ for all $j, k \in n \backslash\{i\}$. To see this, note that $u_{j}=v_{j}$ and $u_{k}=v_{k}$, so by H1, $j \sim k$ iff $u_{j}=u_{k}$ iff $v_{j}=v_{k}$ iff $j \sim^{\prime} k$. We say for short that $\sim$ and $\sim^{\prime}$ agree off of $i$. We cannot say any more about $\sim, \sim^{\prime}$ than that, since we do not know whether $u_{i}=v_{i}$.

What about the $N_{X}$ and $N_{X^{\prime}}^{\prime}$ ? The only sets $S$ of $n-3$ points (the 'black holes') whose representations $h_{S}$ carry information common to both $\left(u_{0}, \ldots\right.$, $\left.u_{n-1}\right)$ and $\left(v_{0}, \ldots, v_{n-1}\right)$ are those $S$ that remain unchanged by moving $u_{i}$. These 'stable sets' are the subsets of $\left\{u_{j}: j \in n \backslash\{i\}\right\}$ of size $n-3$. (They may or may not contain $u_{i}, v_{i}$.)

Now a set $X \in H(\sim)$ corresponds to the black hole $S=\left\{u_{j}: j \in n \backslash X\right\}$. This is a stable set just when it is equal to $\left\{u_{j}: j \in n \backslash(X \cup\{i\})\right\}$. And since $|S|=n-3$, this is exactly when $n \backslash(X \cup\{i\})$ already contains $n-3$ pairwise $\sim$-inequivalent elements. A set $X^{\prime} \in H\left(\sim^{\prime}\right)$ corresponds to this same black hole $S$ just when $X \cup\{i\}=X^{\prime} \cup\{i\}$. For all such $X, X^{\prime}$, we will require $N_{X}(j, k)=N_{X^{\prime}}^{\prime}(j, k)$ for every $j, k \in\left(X \cap X^{\prime}\right) \backslash\{i\}$. Since plainly $X \backslash\{i\}=X^{\prime} \backslash\{i\}$, this is exactly when $N_{X}={ }_{i} N_{X^{\prime}}^{\prime}$. Note here that we do not require that $i \in X \cup X^{\prime}$ - see the definition of $=_{i_{1}, \ldots, i_{k}}$ in section 2.1.

We should therefore demand that $N_{X}={ }_{i} N_{X^{\prime}}^{\prime}$ whenever the above conditions are met.

Well, this all looks very messy, but we are forced into it by our idea, and the notions involved are elementary. Perhaps an example will help.

Example 2. In the notation above, suppose $n=5$, the $\sim$-classes are $\{0\},\{1\}$, $\{2,3\},\{4\}$, and the $\sim^{\prime}$-classes are $\{0,1\},\{2,3\},\{4\}$. See figure 2 . Intuitively, $u_{0}$ is different from all other $u_{j}$, while $v_{0}=v_{1}$. We can see that $\sim$ and $\sim^{\prime}$ agree off of 0 . We have $n-3=2$, and

- $H(\sim)=\{\{0,1\},\{0,2,3\},\{0,4\},\{1,2,3\},\{1,4\},\{2,3,4\}\}$,

- $H\left(\sim^{\prime}\right)=\{\{0,1\},\{2,3\},\{4\}\}$.

Let $i=0$. 
- The sets $X \in H(\sim)$ such that $n \backslash(X \cup\{i\})$ contains $(n-3)=2$ pairwise $\sim$-inequivalent elements are $\{0,1\},\{0,2,3\},\{0,4\}$.

- All sets $X^{\prime} \in H\left(\sim^{\prime}\right)$ are such that $n \backslash\left(X^{\prime} \cup\{i\}\right)$ contains $(n-3) \sim^{\prime}$ inequivalent elements. For example, for $X^{\prime}=\{2,3\}$, we have $n \backslash\left(X^{\prime} \cup\{i\}\right)=$ $n \backslash\{0,2,3\}=\{1,4\}$, and plainly $1 \not{ }^{\prime} 4$.

Of these, the sets $X \in H(\sim)$ and $X^{\prime} \in H\left(\sim^{\prime}\right)$ such that $X \cup\{0\}=X^{\prime} \cup\{0\}$, so they have the same complement in $n$ and correspond to the same 'black hole', are

$$
\begin{aligned}
& -X=X^{\prime}=\{0,1\}, \\
& -X=\{0,2,3\} \text { and } X^{\prime}=\{2,3\}, \\
& -X=\{0,4\} \text { and } X^{\prime}=\{4\} .
\end{aligned}
$$

So for any holograms $\eta=\left(\sim, N_{X}: X \in H(\sim)\right)$ and $\eta^{\prime}=\left(\sim^{\prime}, N_{X^{\prime}}^{\prime}: X^{\prime} \in H\left(\sim^{\prime}\right)\right)$ with $\sim, \sim^{\prime}$ as above, we have $R_{c_{0}}\left(\eta, \eta^{\prime}\right)$ iff $N_{\{0,1\}}={ }_{0} N_{\{0,1\}}^{\prime}, N_{\{0,2,3\}}={ }_{0} N_{\{2,3\}}^{\prime}$, and $N_{\{0,4\}}={ }_{0} N_{\{4\}}^{\prime}$.

The conclusion of this discussion is the following definition.

Definition 3. Let $\mathcal{A}$ be a finite simple relation algebra.

1. Let $\eta=\left(\sim, N_{X}: X \in H(\sim)\right)$ and $\eta^{\prime}=\left(\sim^{\prime}, N_{X}^{\prime}: X \in H\left(\sim^{\prime}\right)\right)$ be $n$ dimensional holograms over $\mathcal{A}$. For each $i, j<n$, define

$-R_{\mathrm{d}_{i j}}(\eta)$ iff $i \sim j$,

- $R_{\mathrm{c}_{i}}\left(\eta, \eta^{\prime}\right)$ iff

(a) $\sim$ and $\sim^{\prime}$ agree off of $i$,

(b) for each $X \in H(\sim)$ and $X^{\prime} \in H\left(\sim^{\prime}\right)$, if $X \cup\{i\}=X^{\prime} \cup\{i\}=I$, say, and $n \backslash I$ contains $n-3$ pairwise $\sim$-inequivalent elements, then $N_{X}={ }_{i} N_{X^{\prime}}^{\prime}$.

This defines a unary relation $R_{\mathrm{d}_{i j}}$ and a binary relation $R_{\mathrm{c}_{i}}$ on the set $M$ of holograms.

2. Let $\mathcal{M}(\mathcal{A})$ be the $n$-dimensional cylindric-type atom structure $\left(M, R_{\mathrm{d}_{i j}}, R_{\mathrm{c}_{i}}\right.$ : $i, j<n)$.

3. Define $\mathcal{C}_{n}(\mathcal{A})=\mathfrak{C m} \mathcal{M}(\mathcal{A})$.

\subsection{Reduction function; undecidability of representability}

Definition 4. Fix a finite non-representable n-dimensional cylindric algebra $\mathcal{C}^{\times}$. Define a function $f$ from finite simple relation algebras to $n$-dimensional cylindric algebras, by

$$
f(\mathcal{A})= \begin{cases}\mathcal{C}_{n}(\mathcal{A}), & \text { if this is an n-dimensional cylindric algebra } \\ \mathcal{C}^{\times}, & \text {otherwise. }\end{cases}
$$


Is the function $f$ a reduction as desired? It is recursive, since $\mathcal{C}_{n}(\mathcal{A})$ is finite and recursively constructible from $\mathcal{A}$, and there is an algorithm to decide whether a finite algebra is an $n$-dimensional cylindric algebra or not. Plainly, $f(\mathcal{A})$ is always an $n$-dimensional cylindric algebra - the use of $\mathcal{C}^{\times}$avoids having to verify that $\mathcal{C}_{n}(\mathcal{A})$ is always a cylindric algebra. Now it can be shown that

$$
\mathcal{A} \text { is representable iff } \mathcal{C}_{n}(\mathcal{A}) \text { is representable. }
$$

We will discuss the proof below. So consider the cases.

1. Suppose that $\mathcal{A}$ is representable. Then $\mathcal{C}_{n}(\mathcal{A})$ is representable, and it follows that $\mathcal{C}_{n}(\mathcal{A})$ is an $n$-dimensional cylindric algebra. The definition of $f$ yields $f(\mathcal{A})=\mathcal{C}_{n}(\mathcal{A})$, and this is representable.

2. Suppose that $\mathcal{A}$ is not representable. There are two possibilities. If $f(\mathcal{A})=$ $\mathcal{C}_{n}(\mathcal{A})$, then by $(3), f(\mathcal{A})$ is not representable. If $f(\mathcal{A})=\mathcal{C}^{\times}$then by choice of $\mathcal{C}^{\times}$it is not representable.

So indeed, modulo (3), $f$ is our desired reduction. Since it is undecidable whether a finite simple relation algebra is representable [2, theorem 18.13], we deduce by Turing reduction that

Theorem 1. For each finite $n \geq 3$, there is no algorithm to decide whether a finite $n$-dimensional cylindric algebra is representable.

For further applications, see [3].

\subsection{Co-representability of $\mathcal{A}$ and $\mathcal{C}_{n}(\mathcal{A})$}

How can we prove (3)? For a full proof, see [3]. We will sketch some of the ideas.

Suppose that $\mathcal{C}_{n}(\mathcal{A})$ is representable. It can be shown that a representation of it over the base set $V$ must be of the form described in section 4.1, with the caveat that the representations $h_{S}$ may be loose (see section 2.1). But if $\mathcal{A}$ has loose representations, it is representable.

Conversely, assume that $\mathcal{A}$ is representable. We need to construct a representation of $\mathcal{C}_{n}(\mathcal{A})$. The key is to construct a $V$ as in section 4.1 in which the $h_{S}$ are 'random' (and loose).

To see why we need randomness, suppose that $\left(u_{0}, \ldots, u_{n-1}\right)$ is an $n$-tuple in $V$ on which the atom $\eta=\left(\sim, N_{X}: X \in H(\sim)\right)$ of $\mathcal{C}_{n}(\mathcal{A})$ 'holds' in the sense of example 1 . Suppose that $R_{\mathrm{c}_{i}}\left(\eta, \eta^{\prime}\right)$, where $\eta^{\prime}=\left(\sim^{\prime}, N_{X}^{\prime}: X \in H\left(\sim^{\prime}\right)\right)$. Then, to be a good representation, there must be some tuple $\left(v_{0}, \ldots, v_{n-1}\right) \in V^{n}$ on which $\eta^{\prime}$ holds, and with $v_{j}=u_{j}$ for each $j \in n \backslash\{i\}$. So there must be a suitable point $v_{i} \in V$.

Why should there be such a point? What are the constraints?

Well, for $\eta^{\prime}$ to hold on $\left(v_{0}, \ldots, v_{n-1}\right)$ given that $\eta$ holds on $\left(u_{0}, \ldots, u_{n-1}\right)$, we require firstly that $v_{i}=v_{j}$ iff $i \sim^{\prime} j$, for each $j \in n \backslash\{i\}$. The case where $i \sim^{\prime} j$ for some such $j$ is easily handled, as it can be shown that $\eta^{\prime}$ already holds 
on $\left(u_{0}, \ldots, u_{i-1}, u_{j}, u_{i+1}, \ldots, u_{n-1}\right)$. So assume that $i \not \chi^{\prime} j$ for every $j \in n \backslash\{i\}$. This means that $v_{i}$ has to be a 'new' element of $V$ not in the set

$$
O=\left\{u_{j}: j \in n \backslash\{i\}\right\}=\left\{v_{j}: j \in n \backslash\{i\}\right\}
$$

of 'old' elements.

Plainly, $|O|<n$. The worst case is when $|O|=n-1$, so let us examine that case. Consider a 'black hole' $S \subseteq O$ of size $n-3$. Bear in mind that there are $\left(\begin{array}{l}n-1 \\ n-3\end{array}\right)$ of these $-\mathcal{O}\left(n^{2}\right)$. Choose $j, k \in n \backslash\{i\}$ such that

$$
O \backslash S=\left\{u_{j}, u_{k}\right\} .
$$

Note that $u_{j}=v_{j}$ and $u_{k}=v_{k}$. Let $X^{\prime}=\{i, j, k\}$. Then $X^{\prime} \in H\left(\sim^{\prime}\right)$. For the hologram $\eta^{\prime}$ to hold on $\left(v_{0}, \ldots, v_{n-1}\right)$, the map $\left(i \mapsto v_{i}, j \mapsto v_{j}, k \mapsto v_{k}\right)$ must be an embedding of $N_{X^{\prime}}^{\prime}$ into $h_{S}$; and we require the analogous property for every $S$.

Can we find such a $v_{i}$ ? Well, let $X=\left\{l<n: u_{l} \notin S\right\}$. Then $j, k \in X \in H(\sim)$. We have $X \cup\{i\}=X^{\prime} \cup\{i\}$, and $n \backslash(X \cup\{i\})$ contains $n-3$ pairwise $\sim$-inequivalent elements, because $\left\{u_{l}: l \in n \backslash(X \cup\{i\})\right\}=S$ and $|S|=n-3$. Since $R_{c_{i}}\left(\eta, \eta^{\prime}\right)$, it follows that $N_{X}={ }_{i} N_{X^{\prime}}^{\prime}$.

Since $\eta$ holds on $\left(u_{0}, \ldots, u_{n-1}\right)$, the map

$$
\left(j \mapsto u_{j}, k \mapsto u_{k}\right)
$$

is a partial embedding of $N_{X}$ into $h_{S}$. But $N_{X}={ }_{i} N_{X^{\prime}}^{\prime}$. So the map

$$
\left(j \mapsto v_{j}, k \mapsto v_{k}\right),
$$

being the exact same map as (4) since $u_{j}=v_{j}$ and $u_{k}=v_{k}$, is also a partial embedding of $N_{X^{\prime}}^{\prime}$ into $h_{S}$.

Now $h_{S}$ is a (loose) representation of $\mathcal{A}$ over $V \backslash S$. By basic properties of relation algebra representations, every $\mathcal{A}$-network with at most 3 nodes embeds homogeneously into every loose representation. So the partial embedding (5) of $N_{X^{\prime}}^{\prime}$ into $h_{S}$ extends to $i$, and we can indeed find a point $v_{i} \in V \backslash S$ such that the map $\left(i \mapsto v_{i}, j \mapsto v_{j}, k \mapsto v_{k}\right)$ is an embedding of $N_{X^{\prime}}^{\prime}$ into $h_{S}$.

It all looks so rosy. But remember: we have found a point $v_{i}$ for this particular $S$. Sure, for each $S \subseteq O$ of size $n-3$, we can find a suitable $v_{i} \in V \backslash S$ in this way: that is, $\forall S \exists v_{i}$. But of course we have to find a single point $v_{i}$ that works for every $S \subseteq O$ of size $n-3$. We need $\exists v_{i} \forall S$.

And that's not all. We have not yet considered the $S \subseteq\left\{v_{0}, \ldots, v_{n-1}\right\}$ with $|S|=n-3$ and $v_{i} \in S$. We must choose $v_{i}$ additionally so that for each of these $S$, if $X^{\prime}=\left\{l \in n \backslash\{i\}: v_{l} \notin S\right\} \in H\left(\sim^{\prime}\right)$, then the map $\left(j \mapsto v_{j}: j \in X^{\prime}\right)$ embeds $N_{X}^{\prime}$ into $h_{S}$.

This seems a tall order. But if the $h_{S}$ are in a sense 'randomly chosen', it is possible to find such a $v_{i}$. Similar arguments can be found in random graph theory and $0-1$ laws for logics.

Actually, the mention of probability is just to give the flavour. We do not really use probability. What we actually do is to build the $h_{S}$ in a kind of forcing 
construction using an infinite game. This ensures that we get the points $v_{i}$ that we need. To do it, it is important that the $h_{S}$ are loose representations of $\mathcal{A}$. For full details, see [3, proposition 4.7].

\section{References}

1. Hirsch, R., Hodkinson, I.: Representability is not decidable for finite relation algebras. Trans. Amer. Math. Soc. 353, 1403-1425 (2001)

2. Hirsch, R., Hodkinson, I.: Relation algebras by games, Studies in Logic and the Foundations of Mathematics, vol. 147. North-Holland, Amsterdam (2002)

3. Hodkinson, I.: A construction of cylindric and polyadic algebras from atomic relation algebras. Algebra Universalis 68, 257-285 (2012)

4. Lyndon, R.: The representation of relational algebras. Annals of Mathematics 51(3), 707-729 (1950)

5. Maddux, R.D.: Topics in relation algebra. Ph.D. thesis, University of California, Berkeley (1978)

6. Maddux, R.D.: Non-finite axiomatizability results for cylindric and relation algebras. J. Symbolic Logic 54(3), 951-974 (1989)

7. Maddux, R.D.: Introductory course on relation algebras, finite-dimensional cylindric algebras, and their interconnections. In: Andréka, H., Monk, J.D., Németi, I. (eds.) Algebraic logic. Colloq. Math. Soc. J. Bolyai, vol. 54, pp. 361-392. NorthHolland, Amsterdam (1991)

8. Monk, J.D.: Studies in cylindric algebra. Ph.D. thesis, University of California, Berkeley (1961)

9. Tarski, A.: On the calculus of relations. J. Symbolic Logic 6, 73-89 (1941)

10. Tarski, A., Givant, S.R.: A formalization of set theory without variables. No. 41 in Colloquium Publications, Amer. Math. Soc., Providence, Rhode Island (1987) 